\title{
NUT midline carcinomas in the thymic region
}

\author{
Yesim Gökmen-Polar ${ }^{1}$, Oscar D Cano ${ }^{1}$, Kenneth A Kesler², Patrick J Loehrer ${ }^{3}$ and \\ Sunil Badve ${ }^{1,3}$ \\ ${ }^{1}$ Departments of Pathology and Laboratory Medicine, Indiana University School of Medicine and Indiana \\ University Simon Cancer Center, Indianapolis, IN, USA; ${ }^{2}$ Department of Surgery, Indiana University School \\ of Medicine and Indiana University Simon Cancer Center, Indianapolis, IN, USA and ${ }^{3}$ Department of \\ Internal Medicine, Indiana University School of Medicine and Indiana University Simon Cancer Center, \\ Indianapolis, IN, USA
}

\begin{abstract}
NUT midline carcinomas (NMCs) are rare tumors described predominantly in the pediatric age group. We recently reported two cases of these tumors occurring in the thymic region. In order to establish the true incidence of these tumors, we examined a large series of thymic carcinomas for morphological features of NUT tumor and further assessed the expression of NUTM1 (also known as NUT) protein by immunohistochemistry. The histological review of slides from 110 cases of thymic carcinoma was undertaken to identify carcinomas with mixed undifferentiated and squamous features that are typically associated with NUT carcinomas. The presenting symptoms, morphological spectrum of tumors and outcome data of patients with these histologies are presented. Immunohistochemistry for NUTM1 was performed on 35 cases of thymic carcinoma with available blocks ( 3 with these histological features and 32 without these features) to exclude the possibility of midline carcinoma. Tumors from 10 patients had features of mixed small cell undifferentiated squamous cell carcinoma (M:F, 1.5:1; age range, 22-79). These patients predominantly presented with advanced disease and had respiratory-related symptoms or chest pain; four had paraneoplastic syndromes. The squamous component in all cases was well differentiated with little or no atypia. The undifferentiated component varied in cell size and lacked characteristic features of small cell carcinoma. All but one patients developed metastases or died within 3 years of diagnosis. NUTM1 expression was seen in two of three tumors with these histological features and in none of the 32 cases without. Mixed small cell undifferentiated carcinomas share histological and immunohistochemical similarity with NMCs and have aggressive clinical course. These tumors are not uncommon and should be considered in the differential diagnosis of carcinomas in the thymic region as novel therapies might be available.
\end{abstract}

Modern Pathology (2014) 27, 1649-1656; doi:10.1038/modpathol.2014.63; published online 23 May 2014

Keywords: mixed small cell carcinoma; NMC; NUT midline lethal carcinoma; thymus neoplasms; undifferentiated carcinoma

Thymic carcinoma is a rare malignant epithelial tumor derived from the thymic gland in the anterior mediastinum. The disease is characterized by cytological atypia and by invasiveness presenting high risk of relapse and death. ${ }^{1-3}$ The current World Health organization (WHO) schema recognizes at least 11 histological subtypes. Combined mixed tumors, like neuroendocrine carcinomathymic carcinoma, have also been reported. ${ }^{4-7}$ Undifferentiated thymic carcinoma is one of the

Correspondence: Dr S Badve, MD, FRCPath, Department of Pathology and Laboratory Medicine, Indiana University School of Medicine and Indiana University Simon Cancer Center, 350 West 11th street, IUHPL 4050, Indianapolis, IN 46202, USA.

E-mail: sbadve@iupui.edu

Received 31 December 2013; revised 27 February 2014; accepted 6

March 2014; published online 23 May 2014 subtypes that can be indistinguishable from other poorly differentiated carcinomas such as nuclear protein in testis (NUT) midline carcinomas (NMCs). ${ }^{8}$ NMCs, also called carcinoma with $\mathrm{t}(15 ; 19)$ translocation, are characterized by chromosomal rearrangement of the NUTM1 gene located on chromosome 15 (commonly called NUT). ${ }^{9}$ NMCs are histologically composed of an admixture of small cell and undifferentiated carcinoma-type tumor cells. The pattern is not dissimilar to that described for a rare variant of thymic carcinoma under the rubric of 'mixed small cell undifferentiated squamous cell carcinoma' by Snover et al. ${ }^{6}$ They described three cases of primary thymic carcinoma, wherein the tumor showed a mixture of welldifferentiated squamous carcinoma with a small cell undifferentiated component. Macroscopically, the tumors were associated with gross evidence of 
invasion and necrosis. Microscopically, the squamous cell component was described as discrete foci of well-differentiated squamous cell carcinoma with focal keratinization and occasional mitotic figures, embedded in a sheet of an undifferentiated component. This undifferentiated component was similar to classical small cell tumor of lung and showed nests of small cells delineated by delicate fibrous septae, scant cytoplasm, finely granular nuclear chromatin, high mitotic count, and extensive areas of necrosis. In one of these three cases, undifferentiated component was described as showing areas where tumor cells were larger, with vesicular nuclei and prominent eosinophilic nucleoli. Delimitation of squamous undifferentiated component was described as abrupt with almost no transition between. Clinically, all three tumors showed an aggressive behavior, unresponsiveness to treatment and associated with fatal outcome in 1-5 years.

More recently, Mukai et $a l^{10}$ published a case of invasive thymic carcinoma with a mixed small cell and squamous cell component. In contrast to cases reported by Snover et al, ${ }^{6}$ the squamous cell component in this case was described as being 'undifferentiated' on histological and immunohistochemical examinations. In spite of aggressive treatment, including chemotherapy, radiotherapy and radical surgery, the patient died 3 years after diagnosis.

The goal of the current study was to identify the incidence of mixed small cell undifferentiated squamous carcinoma of the thymus and examine its relationship with NMCs. Our review identified 10 cases of thymic carcinoma that exhibited this histology. Herein, we describe the presenting symptoms, morphological spectrum and outcomes data of these patients. NUTM1 expression in the tumors was confirmed in two of the three cases on which blocks were available.

\section{Materials and methods}

Following the approval of the Institutional Review Board, we retrospectively reviewed the database at Indiana University. All the cases were diagnosed as malignant thymic neoplasms based on their clinical history, presenting symptoms and findings of imaging and histological studies. All patients were referred to Indiana University Simon Cancer Center for management of their primary or metastatic thymic carcinomas. All available slides were reviewed. Unstained slides or blocks were available for only three cases (in spite of contacting the submitting institutions); this prevented systematic study of the immunohistochemical findings. Immunohistochemistry data, gross description and initial diagnosis, where available, were collected from archival pathology reports from original institution. The possibility of NUT-associated midline carcinoma was assessed by performing immunohistochem- istry for NUTM1 protein (Cell Signaling, Danvers, $\mathrm{MA}$ ) in the three cases with available blocks.

All cases included in this report were selected based on the basis of hematoxylin and eosin-stained morphology. The selection criterion was the presence of two distinct components, namely an undifferentiated cell component and a moderate to well-differentiated squamous cell component, within the same tumor. The undifferentiated component was further classified as small or intermediate or large based on the criteria used in lung tumors. Each case was analyzed for predominant architectural pattern and cell type of the undifferentiated component. The tumors were further classified as having organoid growth pattern defined as nested or trabecular growth pattern with perilobular palisading, and associated with relatively scant stroma; or desmoplastic growth pattern consisting of nest and cords with broad zone of hyaline fibrous stroma, with or without lymphoplasmacytic infiltrate. The percentage of squamous cell component was evaluated as 'low' if presented in less than $50 \%$ of tumor and 'high' if presented in more than $50 \%$ of tumor. Mitotic figures were separately counted in both the components and mitotic activity was classified as high ( $\geq 5$ mitotic figures per 10 high power field (hpf)), moderate ( $>1 / 10$ hpf but less than 5 ), or low $(\leq 1 / 10 \mathrm{hpf})$. Intratumoral lymphocytic infiltrate and percentage of necrosis were also recorded.

The demographic and clinical data collected included age, sex, race, history of smoking, date of initial diagnosis, presenting feature, stage of diagnosis, associated paraneoplastic/autoimmune syndromes, and surgical findings. Details about systemic chemotherapy and radiation therapy were recorded. Follow-up information with respect to development of metastases, site of metastases, and duration of follow-up was extracted from patient charts. Survival information was obtained from charts, tumor registry, and by search of the death registry by social security number and date of birth.

Immunohistochemical analysis for NUTM1 protein was performed on 35 cases where blocks or unstained slides were available ( 3 cases of mixed histology and 32 cases without histological features as control). Staining was performed using the C52 antibody (Cell Signaling) and methods described by French et al. ${ }^{11}$ The NUTM1 (C52B1) rabbit mAb detects endogenous levels of total NUT protein corresponding to the NUTM1 gene (NUT midline carcinoma, family member 1). The antibody also detects endogenous levels of the BRD4-NUT fusion protein present in NUT midline carcinoma, family member 1.

Briefly, 5- $\mu \mathrm{m}$ sections, following deparaffinization and rehydration, were subjected to antigen retrieval in Dako pH 9.0 solution (DakoUSA, Carpinteria, CA) using Decloaking Chamber (BioCare Medical, Walnut Creek, CA). After blocking and quenching endogenous peroxidase activity, sections were incubated with primary rabbit monoclonal anti-NUT antibody, and, after washes, incubated with horseradish 
peroxidase-conjugated secondary antibodies (Envision detection kit, DakoUSA). Staining was developed through incubation with diaminobenzidine, and sections were counterstained with hematoxylin.

\section{Results}

\section{Clinical Findings}

The review identified 10 patients with mixed small cell undifferentiated squamous cell carcinoma; their clinical characteristics are summarized in Table 1. Six of these patients were male (and four female) with an average age at diagnosis of 50.9 years (range, 22-79). Two patients were Caucasians, two African Americans, one Asian, and one Middle Eastern; race information was not available in four patients. One of the patients had a prior history of prostate cancer (Case 9) and another had received radiation as a child for enlarged adenoids (Case 3). Five patients had significant history of smoking. Presenting symptoms were recorded for eight patients; four patients presented only with chest symptoms such as shortness of breath, cough and/or chest pain whereas four presented only with systemic symptoms such as weight loss, fatigue, and lymphadenopathy. Two patients presented with both chest and systemic symptoms. Four patients had symptoms related to autoimmune disease at presentation; one each with Guillain-Barré syndrome, collagen vascular disease (Sjögren syndrome and dermatomyositis), carcinoid-like syndrome, fibromyalgia and pancytopenia. Signs and symptoms suggestive of possible paraneoplastic syndromes documented in the follow-up for four patients included hyponatremia, flushing, pancytopenia, clubbing, skin rash and fibromyalgia.

\section{Stage and Metastases}

In all cases, the tumors were diagnosed as thymic carcinoma. Tumor stage at diagnosis had been recorded for only eight patients. Five were tumor stage IVb, two were stage IVa, and one was stage IIb. Surgical excision was performed in six cases.

Extensive disease, in the form of diffuse pleural dissemination (Case 7) or presence of metastases at diagnosis (five cases), was documented in six cases. Metastatic involvement of two or more sites, including lymph nodes $(n=4)$, brain $(n=2)$, bones $(n=2)$, liver $(n=1)$ and abdominal wall $(n=1)$, was noted in these cases. One patient developed soft tissue metastases 14 months after the initial diagnosis (Case 10).

\section{Therapy Information}

Therapy information was documented in eight cases. Two tumors were unresectable at the time of

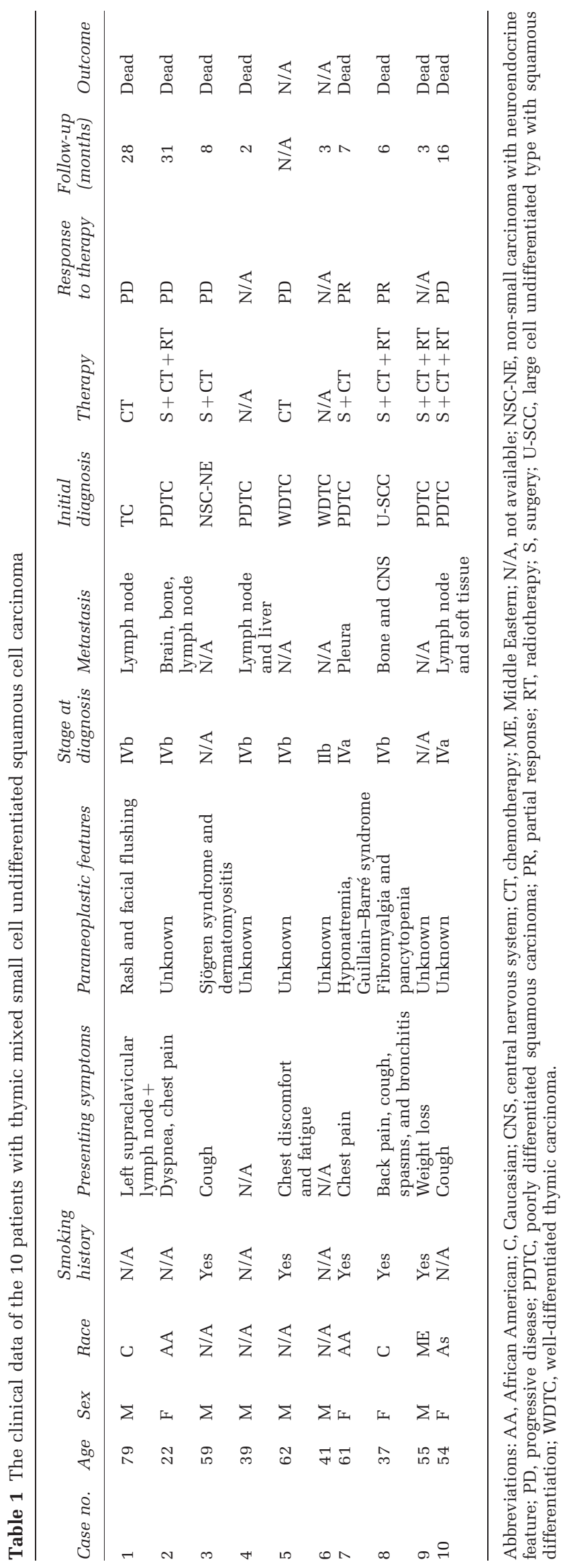


diagnosis and these patients (Cases 1 and 5) received chemotherapy only. Six patients underwent surgery followed by chemotherapy alone (Cases 3 and 7) or by chemotherapy and radiotherapy (Cases 2, 8, 9, and 10). Five patients had limited or no response to therapy and their condition continued progressing. Two patients (Cases 7 and 8) had a partial response ( $>50 \%$ regression) to therapy. For the remaining three patients (Cases 4, 6, and 9), neither the type of therapy nor their response was available.

\section{Survival Information}

In spite of surgical, chemotherapy and radiotherapy treatment, the course of the disease was aggressive with fatal outcome for eight patients ranging from 2 to 31 months following initial diagnosis (median, 7 months). For the remaining two patients, no information regarding their outcomes could be obtained even after searching by social security number.

\section{Pathological Features}

In eight cases, the sections came from the primary tumors: four excisions $(2-8 \mathrm{~cm}$ in size), three vacuum-assisted biopsies, and one needle biopsy. For two patients, only metastatic tissues, supraclavicular lymph node and tumor mass from right ventricular outflow tract, were available. Two cases had slides from both primary and metastatic sites.

The initial pathological diagnosis at the referring institutions was reported as poorly differentiated squamous carcinoma in five cases and well-differentiated carcinoma in two cases. In one case each, thymic carcinoma (not specified), large cell undifferentiated type with squamous differentiation and non-small carcinoma with neuroendocrine feature was recorded.

All 10 tumors were composed of squamous cells and undifferentiated components (Table 2). The transition between the two components was relatively abrupt with the two components being separated by one to three cells of intermediate morphology (Figure 1). The pathological findings, including the number of hematoxylin and eosinstained slides available for each reviewed case (range, 1-14), are summarized in Table 2. Intratumoral necrosis was observed in four cases, although in additional two cases, areas of necrosis were described on gross examination. Nine cases showed desmoplastic growth pattern whereas one case showed organoid growth pattern. Focal scant to moderate intratumoral lymphocytic infiltrate was present in five cases.

The undifferentiated component consisted of sheets of cells arranged in syncytial growth pattern with occasional areas exhibiting a discohesive architecture. In four cases, a mixture of classic small and larger intermediate cells was noted. In most cases, small cells showed speckled chromatin and absence of nucleoli. The larger cell population at times showed vesicular nuclei with a single small nucleolus (Figure 2). Nuclear molding was present in only one case. The cytoplasm, in all cases, was inconspicuous. The mitotic count was evaluated as low in three cases, moderate in one case, and high in six cases.

The squamous cell component occurred as discrete foci embedded in the undifferentiated component of the tumor. In eight cases, this component was in the form of small microscopic foci but, in two cases, it was prominent. Four cases displayed well-developed keratin pearls. Mitotic figures were identified in the squamous component in only two cases.

In one case, the residual tumor, excised post chemotherapy, showed significant response in the mediastinum but showed prominent infiltration of the adjacent lung. In the lung, foci of tumor showed a peribronchial growth and were associated with peripheral palisading. However, glandular spaces or cyst formation, reminiscent of basaloid carcinoma, was not seen.

In two cases, matched primary and metastatic samples were available for review. In these cases,

Table 2 The details of the morphological features of the undifferentiated and squamous cell components are listed below

\begin{tabular}{|c|c|c|c|c|c|c|c|c|}
\hline \multirow[b]{2}{*}{ Case no. } & \multirow[b]{2}{*}{$H \mathcal{H E}$} & \multirow[b]{2}{*}{ Necrosis } & \multicolumn{2}{|c|}{ Squamous component } & \multirow{2}{*}{$\begin{array}{l}\text { Undifferentiated } \\
\text { component } \\
\text { Predominant } \\
\text { cell type }\end{array}$} & \multicolumn{2}{|c|}{ Mitotic activity } & \multirow[b]{2}{*}{$\begin{array}{l}\text { Lymphocytic } \\
\text { infiltrate }\end{array}$} \\
\hline & & & Amount & $\begin{array}{l}\text { Keratin } \\
\text { pearl }\end{array}$ & & $\begin{array}{l}\text { Squamous } \\
\text { component }\end{array}$ & $\begin{array}{l}\text { Undifferentiated } \\
\text { component }\end{array}$ & \\
\hline 1 & 1 & No & Low & Absent & Large & No & High & No \\
\hline 2 & 14 & Yes & Low & Present & Large & Yes & High & No \\
\hline 3 & 1 & Yes & Low & Absent & Large & No & High & No \\
\hline 4 & 2 & No & Low & Present & Large & No & Moderate & No \\
\hline 5 & 5 & Yes & High & Absent & Large & No & Low & Yes \\
\hline 6 & 3 & No & High & Present & Large & No & Low & Yes \\
\hline 7 & 2 & Yes & Low & Absent & Large & No & High & No \\
\hline 8 & 3 & Yes & Low & Present & Small & No & Low & Yes \\
\hline 9 & 1 & Yes & Low & Absent & Large & Yes & High & Yes \\
\hline 10 & 1 & No & Low & Absent & Large & No & High & Yes \\
\hline
\end{tabular}

Abbreviation: H\&E, hematoxylin and eosin. 


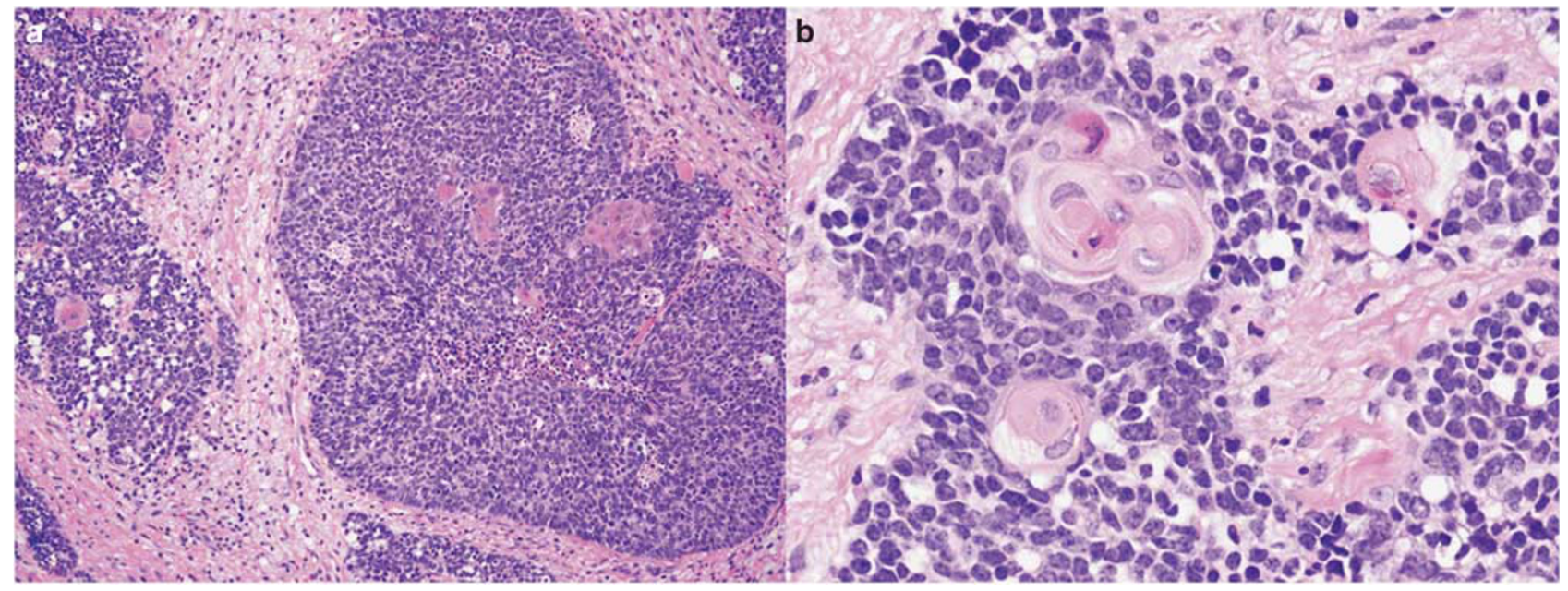

Figure 1 Mixed small cell squamous carcinoma of the thymus. Low and high magnification images (a, b) showing the two components juxtaposed to each other with abrupt transition.

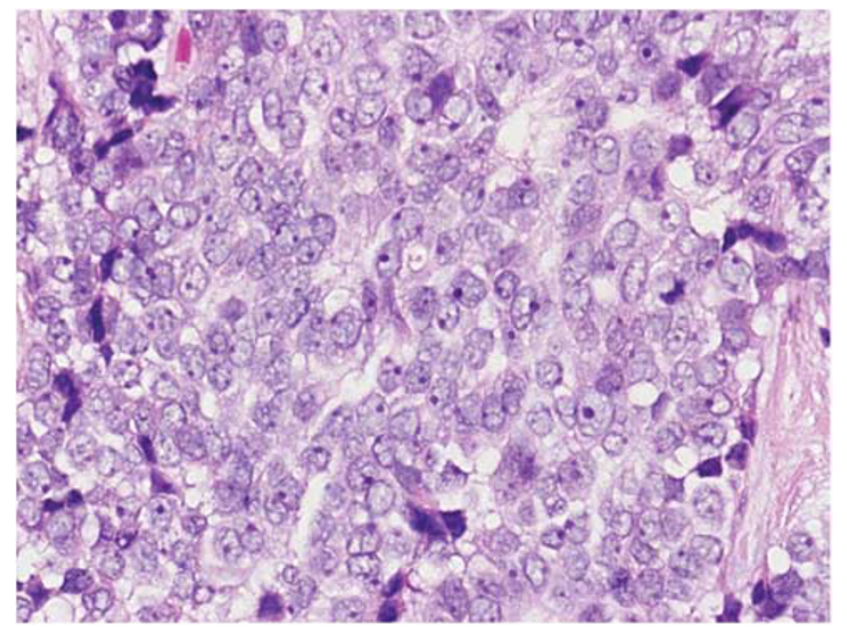

Figure 2 Mixed small cell squamous carcinoma of the thymus. Undifferentiated component is predominant and the cells have vesicular nuclei with small nucleoli and lack the classic molding of small cell carcinoma.

the tumor at both sites had identical morphology (Figure 3).

Immunohistochemistry data were available in seven cases, all tumors were positive for a broad spectrum of cytokeratins (data not shown). Neuroendocrine features (synaptophysin and/or neuronspecific enolase) were also reported as positive in two of the five cases analyzed (Cases 3 and 7). CD5 expression was positive in all three tumors analyzed.

\section{NUTM1 Expression Analysis}

Immunohistochemistry was performed using the NUTM1 antibody (C52; Cell Signaling) generated by French et al. ${ }^{11,12}$ Expression was noted in two of the three cases with blocks (Figure 4a-c).

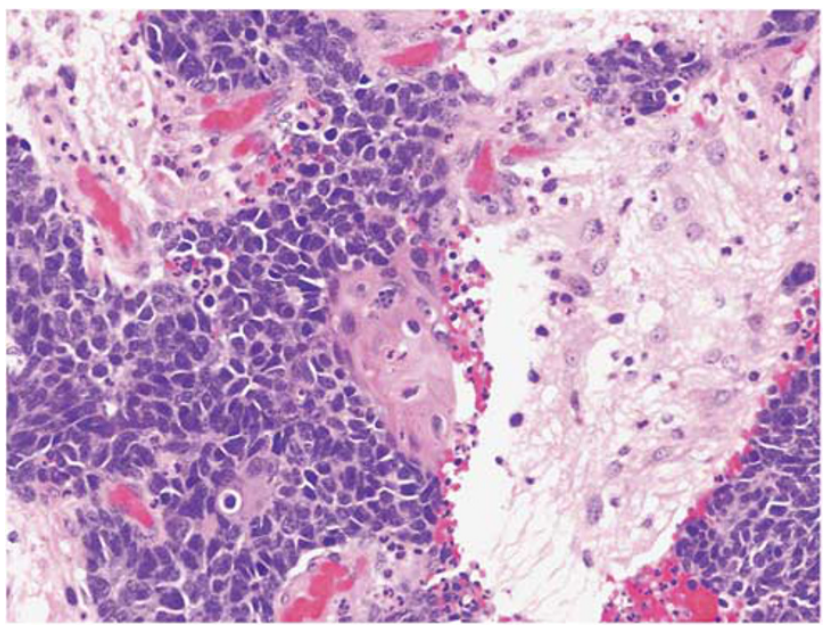

Figure 3 Mixed small cell squamous carcinoma of the thymus. Section is taken from the brain metastases in one of the patients. Note that mixed pattern is retained in the metastases.

Expression was also analyzed in 32 cases without these histological features; none showed expression of NUTM1 protein.

\section{Discussion}

The diagnosis of thymic carcinoma relies heavily on clinical findings, imaging studies and supported by histologic findings. Caution is required in making this diagnosis in the absence of appropriate context. This is especially true when one is dealing with biopsy specimens; in all of the cases presented here, the possibility of extrathymic origin was excluded on clinical and imaging studies. Importantly, in all cases, the diagnosis from the referring institution was thymic carcinoma. 


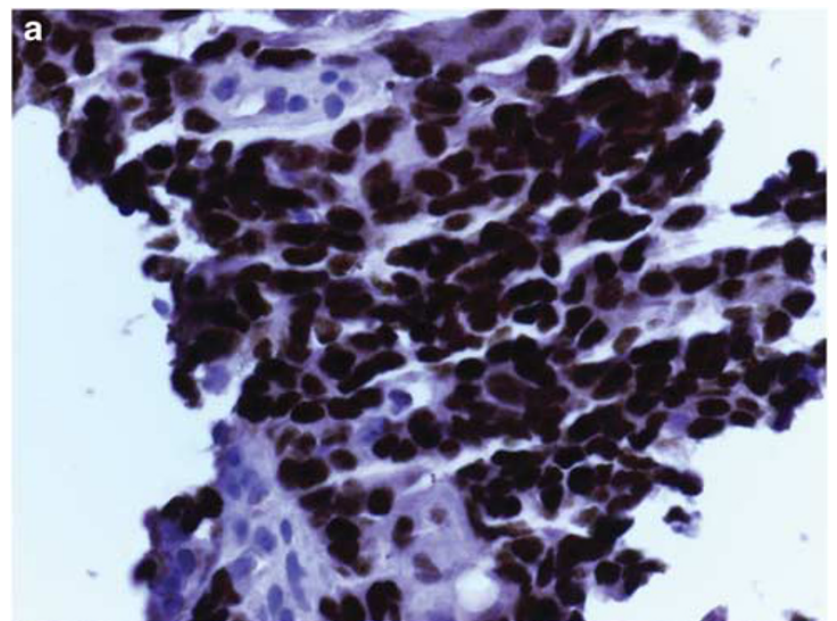

bladder. ${ }^{13,14}$ The older articles focus on whether the undifferentiated component and squamous component had different origins (endodermal versus neuroectoderm).

The characterization of the undifferentiated component is a more difficult issue. The cells, although undifferentiated, lack the classic features of small cell undifferentiated carcinoma seen in the lung. The nuclei are larger and lack nuclear molding. DNA encrustation of stroma and vessels (Azzopardi effect) was not identified in any of the cases. The lack of classic features of small cell carcinoma was also noted in the original description of the entity. ${ }^{6}$ The organoid growth pattern in many cases would suggest a neuroendocrine differentiation. However, only two of the five cases stained for the neuroendocrine markers showed positivity (as per report). In the remainder of the cases, due to lack of unstained material, the neuroendocrine differentiation could not be confirmed. Kuo et $a l^{7}$ studied 22 thymic carcinomas for the presence of neuroendocrine cells and identified them in 4 of 10 squamous cell carcinomas, and in 7 of 8 adenosquamous carcinomas; in almost all cases, this population constituted less than $1 \%$ of the tumor. Wick and Ritter ${ }^{15}$ showed a relatively common occurrence of mixed neuroendocrine cell and squamous cell carcinoma of thymus, postulating the existence of a common thymic epithelial precursor as the progenitor of thymic neuroendocrine cells.

As illustrated in this series, these tumors were most commonly misdiagnosed as some variant of squamous cell carcinoma. Keratinizing variant of squamous cell carcinoma is composed of large polygonal cells arranged in groups and cords, nuclei are vesicular or hyperchromatic, with easily seen nucleoli. Cytoplasm is eosinophilic and keratin pearls, although sometimes focal, are present. Nonkeratinizing squamous cell carcinoma often shows cohesive cellular nests with cells exhibiting distinct cell membranes and at least a moderate degree of cytological atypia. Small cell variant of squamous cell carcinoma tends to exhibit cells with more vacuolated nuclei and visible nucleoli and lack evidence of neuroendocrine differentiation. Basaloid variant of thymic carcinoma tends to have extensive glandular or cyst-like spaces and exhibit peripheral palisading. ${ }^{6,16,17}$ Poorly differentiated squamous cell carcinomas consist of sheets or nests of large polygonal cells with prominent nucleoli and a moderate amphophilic amount of cytoplasm. Small cell neuroendocrine carcinoma is an important consideration, particularly if the squamous component is small and not appreciated. The expression of neuroendocrine markers such as synaptophysin, chromogranin, CD56 and neuronspecific enolase is valuable in the distinction. Entrapped thymic remnants (Hassall corpuscles) within the tumors can be a confounding factor. Primary mixed thymic tumor also has to be carcinomas in the thymus were first described by Snover et $a l^{6}$ and more recently by Mukai et al. ${ }^{10}$ However, tumors with similar morphology have been described in a number of other sites including lung, upper respiratory tract and urinary 
distinguished from a lung primary and metastases from other sites.

The association of these tumors with carcinoma with $t(15 ; 19)$ translocation needs closer examination. ${ }^{17,18}$ Translocation carcinomas can have very similar histology. Recently, these tumors have been shown to have NUTM1 gene fusion; this can be analyzed by cytogenetic studies or specific probes that detect the fusion. ${ }^{12,18}$ More recently, a monoclonal antibody directed against the fusion protein has been generated. We found expression of NUT protein in two of the three cases with available blocks and in none of the 32 cases of thymic carcinomas that lacked these histological features. The NUT antibody is known to be associated with $100 \%$ specificity but relatively low sensitivity $(87 \%) .{ }^{8}$ Given this knowledge, it is very likely that the tumors described herein could be part of the NUT midline tumor family. Classically, NUT tumors have been described in the pediatric age group. However, the International NUT Midline Carcinoma Registry recently presented data on 63 patients with an age range from 0.1-78 years (median, 16 years). ${ }^{9}$ The availability of specific reagents has expanded the age group and recognition of these tumors in adult population. Novel bromodomain inhibitory drugs that specifically target the NUTM1 fusion gene (BRD3-NUT or BRD4-NUT) are in early stages of development. $^{9}$

From a prognostic point of view, the poorly differentiated thymic neuroendocrine cells, combined with squamous cell carcinoma features, have a highly aggressive course, similar to that of classic poorly differentiated neuroendocrine carcinoma (survival time, 1-4 years). ${ }^{19}$ By comparison, the prognosis of thymic squamous cell carcinoma is considerably better than that of neuroendocrine carcinoma types. ${ }^{7,16,20,21}$ NUT midline carcinoma has the highest aggressive course from all of these with fulminant death and average survival time of 18 weeks; the cases described here share many features with NUT tumors. Recently, therapeutic approaches for NMC have been developed involving inhibitors of BRD3 and BRD4 bromodomain (BETi). ${ }^{22-24}$ Two phase I clinical trials with BETi (GSK 525762; Glaxo-Smith Kline and TEN-010; Tensha Therapeutics) (Clinical trials NCT01587703 \& NCT01987362) are ongoing and open to NMC patients. In addition, HDACi demonstrated promising clinical response in pediatric patients. ${ }^{25}$

In summary, we describe a series of 10 malignant tumors from an overall series of 110 cases of thymic carcinoma composed of two distinct components, squamous and undifferentiated, that were characterized by an aggressive course and failure to respond to standard therapies. Given the histological similarity, aggressive behavior, and expression of NUTM1 in at least some cases, we believe that the tumors described herein may represent a form of NUT midline carcinoma. However, the lack of tissue blocks in a number of cases makes it difficult to make a definitive statement in this regard. We recommend that the expression of NUTM1 protein should be analyzed in cases with poorly differentiated or mixed squamous undifferentiated carcinoma histology to exclude the possibility of NUT midline carcinoma.

\section{Disclosure/conflict of interest}

The authors declare no conflict of interest.

\section{References}

1 Rosai J. Histological typing of tumours of the thymus. WHO International Classification of Tumours. Springer: Berlin Heidelberg, 1999.

2 Blumberg D, Burt ME, Bains MS, et al. Thymic carcinoma: current staging does not predict prognosis. J Thorac Cardiovasc Surg 1998;115:303-308; discussion 8-9.

3 Ogawa K, Toita $\mathrm{T}$, Uno $\mathrm{T}$, et al. Treatment and prognosis of thymic carcinoma: a retrospective analysis of 40 cases. Cancer 2002;94:3115-3119.

4 Sensaki K, Aida S, Takagi K, et al. Coexisting undifferentiated thymic carcinoma and thymic carcinoid tumor. Respiration 1993;60:247-249.

5 Paties C, Zangrandi A, Vassallo G, et al. Multidirectional carcinoma of the thymus with neuroendocrine and sarcomatoid components and carcinoid syndrome. Pathol Res Pract 1991;187:170-177.

6 Snover DC, Levine GD, Rosai J. Thymic carcinoma. Five distinctive histological variants. Am J Surg Pathol 1982;6:451-470.

7 Kuo TT, Chang JP, Lin FJ, et al. Thymic carcinomas: histopathological varieties and immunohistochemical study. Am J Surg Pathol 1990;14:24-34.

8 Haack H, Johnson LA, Fry CJ, et al. Diagnosis of NUT midline carcinoma using a NUT-specific monoclonal antibody. Am J Surg Pathol 2009;33:984-991.

9 Bauer DE, Mitchell CM, Strait KM, et al. Clinicopathologic features and long-term outcomes of NUT midline carcinoma. Clin Cancer Res 2012;18:5773-5779.

10 Mukai S, Yao H, Yamamura M, et al. [Thymic carcinoma (mixed small cell undifferentiated squamous cell carcinoma); report of a case]. Kyobu Geka 2003; 56:509-512.

11 French CA, Ramirez CL, Kolmakova J, et al. BRD-NUT oncoproteins: a family of closely related nuclear proteins that block epithelial differentiation and maintain the growth of carcinoma cells. Oncogene 2008;27:2237-2242.

12 French CA. NUT midline carcinoma. Cancer Genet Cytogenet 2010;203:16-20.

13 Barbeaux A, Duck L, Weynand B, et al. Primary combined squamous and small cell carcinoma of the larynx: Report of two cases and discussion of treatment modalities. Eur Arch Otorhinolaryngol 2006;263: 786-790.

14 Pant-Purohit M, Lopez-Beltran A, Montironi R, et al. Small cell carcinoma of the urinary bladder. Histol Histopathol 2010;25:217-221.

15 Wick MR, Ritter JH. Neuroendocrine neoplasms: evolving concepts and terminology. Curr Diagn Pathol 2002;8:102-112. 
16 Suster S, Rosai J. Thymic carcinoma. A clinicopathologic study of 60 cases. Cancer 1991;67:1025-1032.

17 Weissferdt A, Moran CA. Thymic carcinoma associated with multilocular thymic cyst: a clinicopathologic study of 7 cases. Am J Surg Pathol 2011;35: 1074-1079.

18 French CA. Pathogenesis of NUT midline carcinoma. Annu Rev Pathol 2012;7:247-265.

19 Moran CA, Suster S. Thymic neuroendocrine carcinomas with combined features ranging from well-differentiated (carcinoid) to small cell carcinoma. A clinicopathologic and immunohistochemical study of 11 cases. Am J Clin Pathol 2000;113: $345-350$.
20 Hsu CP, Chen CY, Chen CL, et al. Thymic carcinoma. Ten years' experience in twenty patients. J Thorac Cardiovasc Surg 1994;107:615-620.

21 Wick MR, Scheithauer BW, Weiland LH, et al. Primary thymic carcinomas. Am J Surg Pathol 1982;6:613-630.

22 Filippakopoulos P, Qi J, Picaud S, et al. Selective inhibition of BET bromodomains. Nature 2010;468:1067-1073.

23 Schwartz BE, Hofer MD, Lemieux ME, et al. Differentiation of NUT midline carcinoma by epigenomic reprogramming. Cancer Res 2011;71:2686-2696.

24 French CA. NUT midline carcinoma. Nat Rev Cancer 2014;14:149-150.

25 French CA. The importance of diagnosing NUT midline carcinoma. Head Neck Pathol 2013;7:11-16. 